\title{
What Becomes of the Frequent Hospital Attenders with Centrally Mediated Gastrointestinal Pain Syndrome?
}

\author{
Alexander R. Robertson \\ Department of Gastroenterology, Royal Infirmary of Edinburgh, Edinburgh, UK
}

\section{Keywords}

Function gastrointestinal disorders · Centrally mediated gastrointestinal pain syndrome $\cdot$ Abdominal pain . Narcotic bowel syndrome

\begin{abstract}
Objective: Centrally mediated gastrointestinal pain syndrome (CAPS) is an infrequently diagnosed functional condition. A small number of patients do, however, become heavy service users due to difficulty in controlling severe symptoms. We aim to review the outcomes of patients who required frequent hospitalisation with CAPS. Methods: Medical records of patients with multiple CAPS presentations during 2015 ( $n=18$ ) were further analysed and reviewed until January 1st, 2019. Results: Patients (female 15; male 3) had a median age of 33 (IQR 12) years. Gastrointestinal co-diagnosis was common (88.9\%), most frequently irritable bowel syndrome $(50 \%)$, cyclical vomiting syndrome $(27.8 \%)$ or gastro-oesophageal reflux disease (16.7\%). 66.7\% were prescribed regular opiate analgesics. Psychiatric co-morbidity was present in almost all: depression (88.9\%); anxiety/panic disorders (38.9\%) and post-traumatic stress disorder (27.8\%). Social stressors were cited in 33.3\%. $27.8 \%$ were employed, frequently in healthcare. Over the 4-year study period, 18 identified patients accounted for 2,048 nights in hospital
\end{abstract}

and $672 \mathrm{~A}+\mathrm{E}$ attendances. In 2015, these patients spent a median of 19 (IQR 34) nights in hospital each with median hospitalisations of between 7 and 10 nights over the following 3 years. Median A+E attendance was 8 per patient (IQR 8) in 2015, remaining relatively constant between 5 and 6 $\mathrm{A}+\mathrm{E}$ presentations per patient per subsequent year. Conclusion: CAPS is a complicated heterogenous condition resulting from an interplay of physical stimuli with cognitive and emotional factors. As such, management is difficult and requires a multidisciplinary approach with considerable patient ownership of the condition, which is often difficult to achieve.

(c) 2019 S. Karger AG, Basel

\section{Introduction}

More than a third of the population have a function gastrointestinal disorder (FGID) of some form or severity [1]. One of the less frequently encountered of these is centrally mediated gastrointestinal pain syndrome (CAPS), previously known as functional abdominal pain. This is defined as continuous or frequently recurrent abdominal pain, present for at least 6 months, which correlates poorly with intestinal function. To fulfil diagnostic criteria, pain must not be feigned or resulting from other 
organic or functional conditions and must impact on aspects of daily function [2]. The pain is often described by patients as extremely severe and can be expressed in emotional terms affecting a large anatomical area [3].

The conscious perception of gastrointestinal (GI) pain is a complicated process in which visceral input is modulated by other sensory, cognitive and emotional factors [4, 5]. Unlike irritable bowel syndrome (IBS), intestinal features are largely disconnected in CAPS, and it seems cognitive and emotional features play a larger role in the pain experienced [2]. Personality and mood in adulthood are affected and altered by childhood experiences, with physical or sexual abuse during childhood linked to such disorders. Abuse increases the incidence of functional GI disorders in adulthood [6] and is overrepresented in patients with CAPS [7]. Prospectively followed up, those with higher anxiety scores more frequently developed FGID [8].

As a small subset of patients with severe and debilitating FGID, those with CAPS have a comparatively high rate of health service use [9]. Unfortunately, as a complicated and heterogenous disorder, CAPS is often poorly understood. Possibly due to a lack of training in the area, some gastroenterologists do not feel comfortable managing patients with complicated functional disease [10]. The wide differential for abdominal pain and a lack of definitive testing for CAPS often leads to over-investigation, in part due to a lack of confidence in the diagnosis for both physician and patient with the fear that an alternative pathology is being missed [11].

A need for a good physician-patient relationship with a multidisciplinary approach is repeatedly highlighted [2, 12], but this can be difficult to accomplish. Achieving patient engagement and ownership of their condition is vital but can be hampered by complex psychiatric issues or personality disorders [10]. Patients seeking medical help are often unsatisfied by the response and care they receive [13] with many feeling that their concerns were not resolved by the diagnosis or explanation given by the physician [14]. It is not uncommon for patients to place emphasis on the physical symptoms and feel that discussion of emotional factors is invalidating their primary complaint, the pain. In this way, some patients place high expectations on healthcare teams and seek ongoing investigations or narcotics to validate the "realness" of their pain [3].

Although the majority of individuals with CAPS are successfully managed in a community setting, there is a subset who repeatedly present to hospital with abdominal pain. These patients represent a small group but are difficult to manage, often due to severe and treatment refractory symptoms with complicated psychosocial factors.

This retrospective observational study aims to characterise patients admitted with CAPS, their short-term follow-up and outcomes. We seek to establish if "missed" pathology emerged over subsequent follow-up and whether with time these patients managed to spend more time out of hospital, with fewer emergency presentations.

\section{Materials and Methods}

To isolate patients with multiple presentations due to functional abdominal pain, suggestive of CAPS, hospital admissions across a large NHS trust in the South East of Scotland were screened for inclusion (Fig. 1). 2,253 admissions during the 2015 calendar year were coded as "functional intestinal disorder, unspecified" (K59.9) or "other and unspecified abdominal pain" (R10.4). Three hundred of these presentations were patients who presented $\geq 2$ times with abdominal pain, and these patients were further screened for the likely cause of their presentations.

Clinical information was gathered through the hospital healthcare system (TrakCare, ${ }^{\circ}$ Intersystems, Cambridge, MA, USA) with data extracted and analysed utilising Microsoft Excel $\left({ }^{\circ} \mathrm{Mi}\right.$ crosoft, Redmond, WA, USA). Thirty patients with multiple admissions fulfilled criteria for CAPS. Although CAPS was a likely contributing factor to their admissions, 12 of these patients were subsequently excluded as a separate precipitant to their pain was identifiable. These included pregnancy, pancreatitis, adenomyosis, gastroparesis, IBS-C (constipation-predominant IBS) and endometriosis, all on a background of possible CAPS. The 18 remaining patients were further analysed and records reviewed until January 1 st, 2019.

\section{Results}

The 18 patients with $\geq 2$ admissions during 2015 with CAPS (female 15; male 3) had a median age of 33 (range 25-55 and IQR 12) years of age. Each of these patients had at least 1 previous presentation with abdominal pain and had been given variable diagnoses following suitable investigations but best fulfilled diagnostic criteria for CAPS [2].

\section{Co-Morbidity}

$16 / 18(88.9 \%)$ had at least 1 additional GI co-diagnosis (Table 1). The most common of these was IBS-C (+/slow-transit constipation or anorectal dysfunction) in $8 / 18$ (44.4\%) with 1 further having a diagnosis of IBS-D (diarrhoea-predominant IBS). 5/18 (27.8\%) of the patients had been diagnosed with cyclical vomiting syndrome and 3/18 (16.7\%) with gastro-oesophageal reflux disease. Other GI co-morbidities listed were gastroparesis $(n=1)$, small intestinal bacterial overgrowth (SIBO) $(n=$ $1)$ and peptic ulcer disease $(n=1)$.

$12 / 18(66.7 \%)$ of the patients were prescribed regular opiate analgesics. $8 / 18(44.4 \%)$ of the patients were prescribed regular tricyclic antidepressants and 8/18 (44.4\%) serotonin-norepinephrine reuptake inhibitors or selective serotonin reuptake inhibitors at some point during 2015. 
Fig. 1. Study inclusions/exclusions. CAPS, centrally mediated gastrointestinal pain syndrome; CVS, cyclical vomiting syndrome; IBS, irritable bowel syndrome.
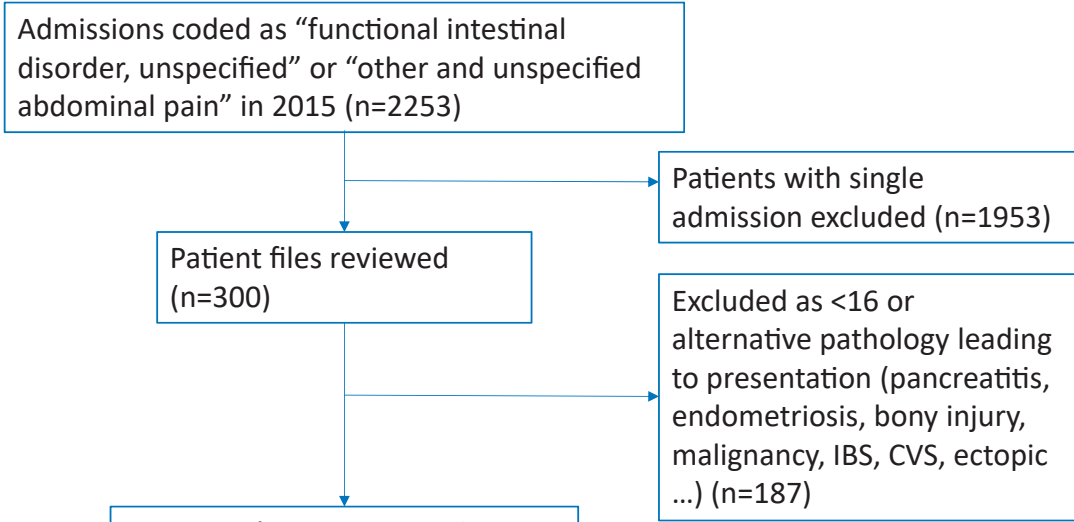

113 attendances in 30 patients suggestive of CAPS

Individuals with $\geq 2$ admissions due to CAPS

in 2015 included in final analysis $(n=18)$

Table 1. GI and psychiatric co-morbidity

\begin{tabular}{llll}
\hline Gastrointestinal co-diagnosis & Prevalence $(n)$ & Psychiatric co-diagnosis & Prevalence $(n)$ \\
\hline IBS-C (+/- slow-transit constipation or anorectal dysfunction) & $44.4 \%(8)$ & Depression & $88.9 \%(16)$ \\
CVS & $27.8 \%(5)$ & Anxiety/panic disorders & $38.9 \%(7)$ \\
GORD & $16.7 \%(3)$ & PTSD & $27.8 \%(5)$ \\
SIBO & $5.6 \%(1)$ & Social stressors & $33.3 \%(6)$ \\
Gastroparesis & $5.6 \%(1)$ & Schizophrenia & $5.6 \%(1)$ \\
IBS-D & $5.6 \%(1)$ & Emotionally unstable PD & $5.6 \%(1)$ \\
PUD & $5.6 \%(1)$ & & \\
Nil & $5.6 \%(1)$ & &
\end{tabular}

IBS-C, irritable bowel syndrome-constipation predominant; CVS, cyclical vomiting syndrome; GORD, gastro-oesophageal reflux disease; SIBO, small intestinal bacterial overgrowth; IBS-D, irritable bowel syndrome-diarrhoea predominant; PTSD, post-traumatic stress disorder; $\mathrm{PD}$, personality disorder.

Psychiatric comorbidity was almost universal in this group with most of the 18 patients in this cohort having multiple psychiatric diagnoses (Table 1). Self-harm and substance dependence were also a common feature. 16/18 (88.9\%) had a diagnosis of depression, most frequently as a diagnosis made in primary care prior to presentations with abdominal pain. 1 of the patients without a diagnosis of depression had schizophrenia. Anxiety and panic disorders were also common, seen in 7/18 (38.9\%), and 5/18 $(27.8 \%)$ had a diagnosis of post-traumatic stress disorder. Social stressors in the lead-up to admission with pain were cited in 6 of the patients (33.3\%) but seem to have been poorly investigated and, as such, are likely to have been underreported. There was 1 patient diagnosed with emotional unstable personality disorder and 1 with widespread chronic pain syndrome.
$5 / 18(27.8 \%)$ of the patients were either in employment or education during 2015: carer $(n=2)$, dental nurse $(n=1)$, pharmacist $(n=1)$ and student $(n=1)$. The remaining 13/18 (72.2\%) were either unemployed or on long-term sick leave from their place of work.

Inpatient management was variable, with admissions under gastroenterology, general medicine, general surgery, gynaecology and psychiatry teams. During 2015, 17/18 (94.4\%) had input from a psychiatrist and 15/18 (83.3\%) input from a gastroenterologist; and in most of these cases, this was a GI consultant with a specialist interest in functional disorders.

\section{Number of Nights in Hospital}

Admission to inpatient services occurred via self-presentation to $\mathrm{A}+\mathrm{E}$, following referral direct from primary 


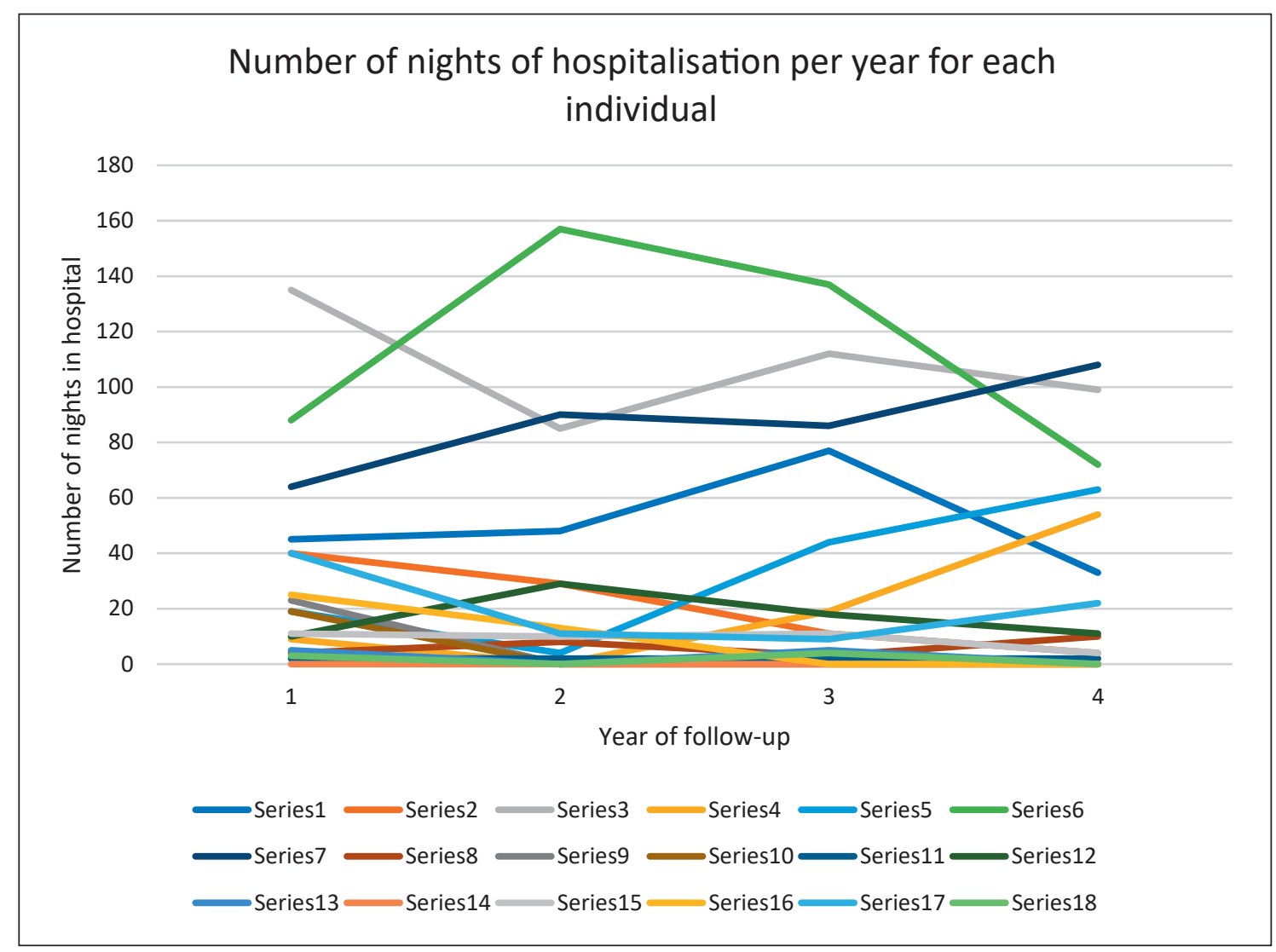

Fig. 2. The number of nights spent in hospital by each individual by year.

care or from a specialty clinic. In 2015, these 18 patients spent a total of 542 nights in hospital with a median of 19 nights per patient (range $0-135$ and IQR 34). This remained relatively constant over the following 3 years with a median hospitalisation of 9 nights (IQR 29) in 2016, 10 nights (IQR 36) in 2017 and 7 nights (IQR 49) in 2018 (Fig. 2).

Over the 4-year study period, the 18 patients accounted for a total of 2,048 nights in hospital with the most, 454 nights, for 1 individual. The least nights spent in hospital was 0 over the 4 -year period; this patient did attend $\mathrm{A}+\mathrm{E} 37$ times, however, but preferred to leave following analgesia.

\section{Number of $A+E$ Attendances}

In 2015 , the number of $A+E$ attendances ranged from 0 to 30 , with a median number of attendances of 8 per patient (IQR 8). Over subsequent years, this level remained relatively constant with median presentations of 5 (IQR 8) in 2016, 6 (IQR 9) in 2017 and 5 (IQR 7) in 2018 (Fig. 3).

\section{Conclusions}

Functional GI disorders are extremely common in the general population, but CAPS is one of the less frequently encountered. Although for many patients CAPS is suc- cessfully managed day to day and then as an outpatient during flares, there are patients who frequently attend hospital as they suffer with symptoms. Non-specialists can often struggle with these patients and become frustrated by their repeat attendances and lack of response to treatment.

CAPS is a complicated heterogenous condition resulting from an interplay of physical stimuli with cognitive and emotional factors. Review of the cohort seen here would suggest that there are different degrees to which physical and emotional factors influence a patient's symptoms and subsequent behaviour.

Nine of 18 patients (50\%) had a co-diagnosis of IBS; most commonly constipation predominant. This would support a theory in which the pathogenesis of CAPS could relate to an extreme of the IBS spectrum where pain predominates with intestinal features less problematic to the patient [2]. In those without IBS, upper GI functional disorders were common, cyclical vomiting syndrome being the most prevalent of these.

As in painful IBS [10], tricyclic antidepressants are recommended as a mainstay of therapy. Serotonin-norepinephrine reuptake inhibitors or selective serotonin reuptake inhibitors or even atypical antipsychotics are alternatives, but opiates are considered to be contraindicated 


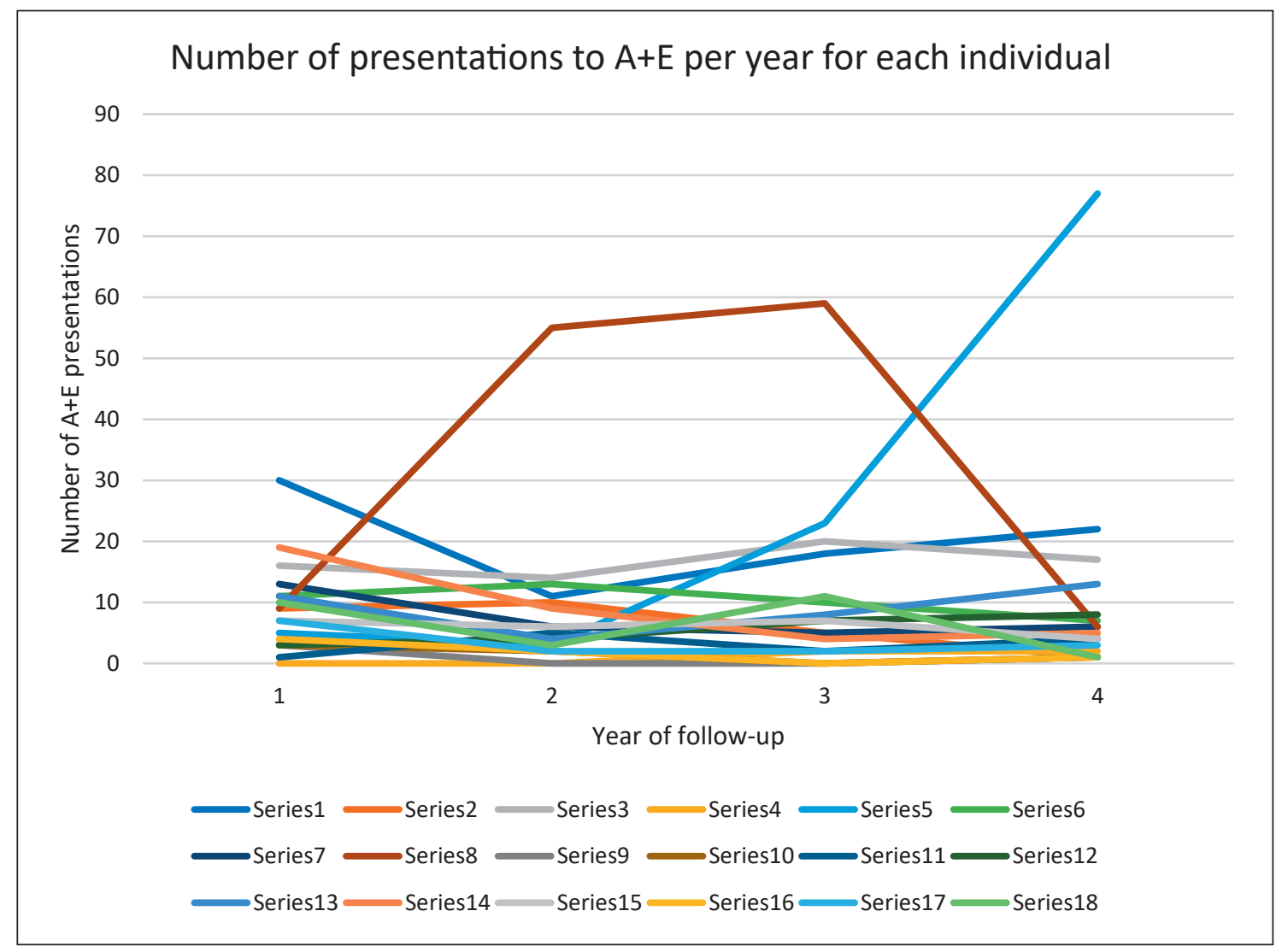

Fig. 3. The number of presentations to $A+E$ by each individual by year.

due to a risk of narcotic bowel syndrome, addiction and GI side effects [2]. Unfortunately, it can be seen that patients with functional pain syndromes are often prescribed opiates as they are desperate for relief and clinicians struggle to control their symptoms. In non-specialist hands, the default approach remains the WHO pain ladder method of escalation, which in this often pharmacologically resistant group can rapidly escalate to high doses of narcotics [15]. In this population, 12 of the 18 (66.7\%) patients were prescribed regular opiates resulting in an overlay of opiate-related bowel disruption, painful constipation or even narcotic bowel syndrome. This greatly complicates the management of CAPS patients and to some leads to a perception of "opiate seeking."

The psychiatric component in those presenting during the study period was clear with $16 / 18$ (88.9\%) diagnosed with depression, $7 / 18$ (38.9\%) with anxiety and panic disorders, 5/18 (27.8\%) with post-traumatic stress disorder and 6/18 (33.3\%) having clear social stressors documented during admission. Due to the retrospective nature of this study, it is impossible to know whether CAPS is secondary to these psychiatric morbidities or whether chronic pain results in psychological damage to these young adults. In those with ongoing symptoms, it is important to have psychiatric input and consider interventions such as cognitive behavioural therapy, mindfulness, psychodynamic interpersonal psychotherapy or hypnotherapy, but to fully address psychosocial issues, a multidisciplinary approach is often required. In paediatric practice, there is evidence for significant improvement in abdominal pain with hypnotherapy, cognitive behavioural therapy and probiotics (Lactobacillus rhamnosus GG and VSL\#3), but there is little evidence for this in adults $[2,10,16]$.

Of interest, one of the only patients to display a sustained decrease in both length of stay and number of $\mathrm{A}+\mathrm{E}$ attendances was also treated for SIBO. Although this individual suffered from other functional disorders and psychiatric issues, the decrease in service use could suggest that her abdominal pain was ameliorated with treatment of SIBO and not an improvement in CAPS. With a high prevalence of GI co-morbidities, a combination of factors, not necessarily all centrally mediated, are likely to be at play in some of this group of patients.

There are several weaknesses in this study design. A small sample size and relatively short follow-up prevents a full understanding of what is an underdiagnosed and complex long-term condition. The data is observational and retrospective. The recording of total number of $\mathrm{A}+\mathrm{E}$ attendances and nights in hospital was intended to estimate service use and, therefore, impact on individuals, 
but this may include presentations with alternative pathology, not limited to their CAPS, over the follow-up period. Patients with repeat presentations were selected (Fig. 1) as they are the most frequently encountered by inpatient teams.

It can be seen that those patients presenting in 2015 continue to be heavy service users. Over the 4 -year period, these 18 patients accounted for $672 \mathrm{~A}+\mathrm{E}$ attendances. The highest number of attendances by an individual during a calendar year was 77, and 1 patient spent 157 nights in hospital over a year (2016), evidencing the severity of the symptoms and difficulty in managing them.

The cohort seen were all young adults of working age, yet only 5/18 (27.8\%) remained in employment or education. Interestingly, healthcare professions predominated with the group, including 2 carers, 1 pharmacist and 1 nurse.

The aim of this study was to investigate how those requiring repeated admission with CAPS during 2015 developed over the subsequent 3 years. $\mathrm{A}+\mathrm{E}$ attendances and overnight hospital admissions were taken as markers of poorly controlled CAPS, and we would suggest that the trends seen here indicate that for many of those with repeated presentations and ongoing symptoms there is little or no resolution over the subsequent 3 years. Repeated attendances at $\mathrm{A}+\mathrm{E}$ and hospitalisation of these young adults would suggest that care is often difficult and suboptimal, with care being split between medical, GI, gynaecology, surgical and psychiatric services without standardisation or development of specialised multidisciplinary care. A difficulty in achieving this standardisation is the heterogeneity of the condition and those who present with it.

\section{Acknowledgements}

With thanks to Drs K. Trimble and W. Wojcik of the Royal Infirmary of Edinburgh for providing advice and critical review of the manuscript prior to preparation of the final draft.

\section{Statement of Ethics}

This study was performed as a service evaluation using routinely collected anonymised data and, as such, no ethics committee review was required.

\section{Disclosure Statement}

The authors have no competing interests to declare.

\section{Funding Sources}

No funding was received.

\section{Author Contributions}

A.R.R. designed the study, collated and analysed the data and prepared the final manuscript.

\section{Data Availability}

The data that support the finding of this study are available on request from the corresponding author, A.R.R. The data are not publicly available due to their containing information that could compromise the privacy of research participants.

\section{References}

1 Koloski NA, Talley NJ, Boyce PM. Epidemiology and health care seeking in the functiona GI disorders: a population-based study. Am J Gastroenterol. 2002 Sep;97(9):2290-9.

2 Keefer L, Drossman DA, Guthrie E, Simrén M, Tillisch K, Olden K, et al. Centrally mediated disorders of gastrointestinal pain. Gastroenterology. 2016 Feb;S0016-5085(16)00225-0.

3 Thompson WG, Longstreth GF, Drossman DA, Heaton KW, Irvine EJ, Müller-Lissner SA. Functional bowel disorders and functional abdominal pain. Gut. 1999 Sep;45 Suppl 2:II43-7.

4 McGrath PA. Psychological aspects of pain perception. Arch Oral Biol. 1994;39 Suppl: 55S-62S.

5 Shankland WE 2nd. Factors that affect pain behavior. Cranio. 2011 Apr;29(2):144-154.

6 Jones MP, Oudenhove LV, Koloski N, Tack J, Talley NJ. Early life factors initiate a 'vicious circle' of affective and gastrointestinal symptoms: A longitudinal study. United European Gastroenterol. 2013 Oct;1(15):394-402.
7 Drossman DA. Abuse, trauma, and GI illness: is there a link? Am J Gastroenterol. 2011 Jan; 106(1):14-25.

8 Koloski NA, Jones M, Kalantar J, Weltman M, Zaguirre J, Talley NJ. The brain - gut pathway in functional gastrointestinal disorders is bidirectional: a 12-year prospective population-based study. Gut. 2012 Sep;61(9):128490.

9 Maxton D, Whorwell P. Use of medical resources and attitudes to health care of patients with "chronic abdominal pain". Br J Med Econ. 1992;2:75-9.

10 Spiller R, Aziz Q, Creed F, Emmanuel A, Houghton L, Hungin P, et al.; Clinical Services Committee of The British Society of Gastroenterology. Guidelines on the irritable bowel syndrome: mechanisms and practical management. Gut. 2007 Dec;56(12):1770-98.

11 Drossman DA. Chronic functional abdominal pain. Am J Gastroenterol. 1996 Nov; 91(11):2270-81.
12 Clouse RE, Mayer EA, Aziz Q, Drossman DA Dumitrascu DL, Mönnikes H, et al. Functional abdominal pain syndrome. Gastroenterology. 2006 Apr;130(5):1492-7.

13 Drossman DA, Morris CB, Schneck S, Hu YJ, Norton NJ, Norton WF, et al. International survey of patients with IBS: symptom features and their severity, health status, treatments, and risk taking to achieve clinical benefit. J Clin Gastroenterol. 2009 Jul;43(6):54150.

14 Thompson WG, Heaton KW, Smyth GT, Smyth C. Irritable bowel syndrome in general practice: prevalence, characteristics, and referral. Gut. 2000 Jan;46(1):78-82.

15 World Health Organisation (WHO). WHO's pain ladder. WHO; 2012.

16 Rutten JM, Korterink JJ, Venmans LM, Benninga MA, Tabbers MM. Nonpharmacologic treatment of functional abdominal pain disorders: a systematic review. Pediatrics. 2015 Mar;135(3):522-35. 